\title{
ASPECTOS MORFOGÊNICOS NO DESENVOLVIMENTO INICIAL DA CANA-DE-AÇÚCAR RB 867515 IRRIGADA
}

\author{
Catariny Cabral Aleman ${ }^{1}$, Dario Sousa ${ }^{2}$ \\ ${ }^{1}$ Universidade Federal de Viçosa - UFV, Departamento de Engenharia Agrícola, Viçosa, MG. ${ }^{2}$ Universidade do Oeste Paulista - \\ UNOESTE, Presidente Prudente, SP. E-mail: catariny@ufv.br
}

\section{RESUMO}

A cana-de-açúcar apresenta ampla área cultivada no estado de São Paulo. Em decorrência das precipitações desuniformes e heterogêneas ao longo do ano, torna-se importante o estudo da irrigação afim de definir um correto manejo hídrico da cultura. A definição da demanda hídrica nas diferentes fases de desenvolvimento da cultura, favorece o desenvolvimento morfofisiológico. O objetivo do presente trabalho foi avaliar parâmetros morfogênicos da cana-de-açúcar RB 867515 irrigada durante o desenvolvimento inicial. O experimento foi conduzido em vasos de PVC no campus II da UNOESTE, durante a fase inicial de desenvolvimento vegetativo da cana-de-açúcar. Os tratamentos foram compostos por diferentes lâminas de irrigação (50, 75, 100, 125 e 150\% da evapotranspiração da cultura - ETc). As lâminas de irrigação foram calculadas pelo método de Penmam-Monteith recomendado pela FAO. O delineamento experimental se deu em blocos ao acaso,sendo 5 lâminas de irrigação e 5 blocos. Avaliou-se: altura quinzenal das plantas (AP), número de folhas quinzenal (NF), massa seca de parte aérea (MSPA), eficiência da água (EUA), comprimento de raiz (CR) e matéria seca de raiz (MSR). Os parâmetros obtidos foram submetidos a ANOVA e posteriormente ao Teste de Scott-Knott ao nível de probabilidade 5\% para comparação de médias. Para os parâmetros biométricos não houve diferença entre as médias obtidas. A lâmina de $50 \%$ ETc foi a que apresentou maior eficiência do uso da água. Considerando os parâmetros morfogênico não houve diferenças estatísticas para as lâminas de irrigação estudadas. No entanto, avaliando a relação entre massa seca e lâmina de irrigação aplicada, concluiu-se que $50 \%$ ETc resultou na maior eficiência do uso da água.

Palavras-chave: eficiência do uso da água; manejo de irrigação; necessidade hídrica; Saccharum sp.

\section{MORPHOGENIC ASPECTS IN THE INITIAL DEVELOPMENT OF SUGARCANE RB 867515 IRRIGATED}

\begin{abstract}
The sugar cane presents extensive acreage in the State of São Paulo in Brazil. Due to the desuniform and heterogeneous rainfall throughout the year, it is important to the study of irrigation in order to set a correct water management culture. The definition of water demand in the different stages of development of culture, promotes the development morphophisiological. The purpose of this study was to evaluate parameters of morphogenesis of sugar cane RB 867515 irrigated during the initial development. The experiment was conducted in PVC pots on campus II UNOESTE, during the initial phase of vegetative development of sugarcane. The treatments were composed of different irrigation blades $(50,75,100,125$ and 150\% of evapotranspiration of culture - ETc). The depths of irrigation were calculated by the method of Penmam-Monteith recommended by FAO. The experimental design in randomized blocks, with 5 depths of irrigation and 5 blocks. Assessed: biweekly height plants (AP), sheet number bi-weekly (NC), dry mass of the shoot (MSPA), water efficiency (USA), length of root (CR) and root dry matter (MSR). The parameters obtained were submitted to ANOVA and later by Scott-Knott test at 5 probability level for comparison of averages. For the biometric parameters there were no differences between the means obtained. The depth of irrigation of $50 \%$ ETc was presented the greater efficiency of water use. Considering the parameters morfogênico there was no statistical differences for studied irrigation. However, assessing the relationship between blade and dry mass of irrigation applied, it was concluded that $50 \%$ ETc resulted in greater efficiency of water use.
\end{abstract}

Keywords: efficiency of water use; irrigation management; water requirements; Saccharum sp.

\section{INTRODUÇÃO}

A demanda pelos produtos do setor sucroalcooleiro está crescendo e mais da metade do açúcar comercializado no mundo é produzido pelo Brasil. Na safra 2014/2015 a produtividade agrícola foi de 90,66 toneladas por hectare para cana de $1^{\circ}$ corte (CONAB, 2014). Para as exportações, o volume previsto para 2019 é de 32,6 milhões de tonelada (BRASIL. Ministério da Agricultura, 2014).

A cana-de-açúcar é uma planta que pertence ao gênero Saccharum $s p$. As espécies de 
cana-de-açúcar são provenientes do sudeste asiático. A planta é a principal matéria-prima para a fabricação do açúcar e álcool (etanol), caracterizada pelo perfilhamento de maneira abundante durante o desenvolvimento inicial (MAGRO et al., 2011).

A disponibilidade hídrica do solo é um dos fatores ambientais que mais influenciam a produção da cana-de-açúcar, pois determina o estabelecimento da cultura durante o estádio vegetativo (RAMESH, 2000). Para o uso eficiente de água pela cana-de-açúcar, é fundamental identificar a necessidade hídrica da cultura para obtenção de produções ótimas (WIEDENFELD; ENCISO, 2008). A distribuição irregular e a redução dos volumes de chuvas durante 0 crescimento vegetativo da cultura ou períodos prolongados com baixa disponibilidade hídrica afetam negativamente a qualidade tecnológica da cultura, produtividade do colmo, na produção fitomassa e no rendimento de sacarose (OLIVEIRA et al., 2011).

Dantas Netto et al. (2006) e Farias et al. (2008) afirmaram que para a obtenção de produtividade elevada atingindo o potencial genético da cultura, o uso tecnológico de irrigação é imprescindível. A determinação da quantidade de água necessária para a irrigação é um dos principais parâmetros para o correto planejamento, dimensionamento e manejo de qualquer sistema de irrigação, bem como para avaliação de recursos hídricos (DAMIÃO et al., 2010). A necessidade de água a ser aplicada por irrigação para satisfazer às demandas das culturas é estimada através do balanço das entradas (chuva) e das saídas (evapotranspiração) e a capacidade de armazenamento da água no solo, função do tipo de solo e da profundidade efetiva do sistema radicular da cultura, sendo então 0 balanço hídrico 0 nome desta contabilidade (PEREIRA et al., 2002).

Em virtude da distribuição irregular da precipitação e da necessidade de determinar uma lâmina de irrigação ótima para a produção de cana-de-açúcar, o objetivo do presente trabalho foi avaliar parâmetros morfogênicos de cana-deaçúcar RB $86 \quad 7515$ irrigada durante o desenvolvimento inicial.

\section{MATERIAL E MÉTODOS}

O experimento foi conduzido em casa de vegetação com cobertura telada e sobre bancada na área experimental no campus II da Universidade do Oeste Paulista, Presidente Prudente - SP. O experimento teve duração de 2,5 meses no ano de 2014, com início em 29 de abril e término em16 de julho. Adotou-se o delineamento experimental em blocos casualizados, com cinco lâminas de irrigação aleatorizadas e cinco blocos, totalizando 25 unidades experimentais.

Inicialmente, foram preparadas as mudas da cana-de-açúcar RB 867515 em recipientes plásticos de $200 \mathrm{~mL}$ preenchidos com solo peneirado. Após 40 dias do plantio (DAP), as mudas foram transplantadas para vasos de PVC com dimensões de $20 \mathrm{~cm}$ de diâmetro $\times 20 \mathrm{~cm}$ de altura. Os vasos foram preenchidos com um $X$ $\mathrm{dm}^{3}$ de solo corrigido nutricionalmente de acordo com a recomendação de Segato et al. (2006) para a cultura da cana-de-açúcar (Tabela 01).

Durante o período de sete dias após o transplantio o solo foi mantido em capacidade de campo, afim de garantir o melhor pegamento das mudas. Após este período iniciou a aplicação diferenciada das lâminas de irrigação.

Tabela 1. Análise química do solo.

\begin{tabular}{|c|c|c|c|c|c|c|c|c|c|c|c|c|}
\hline $\begin{array}{c}\mathrm{pH} \\
\left(\mathrm{CaCl}_{2}\right)\end{array}$ & $\begin{array}{l}\text { M.O. } \\
\mathrm{g} \mathrm{dm}^{-3}\end{array}$ & $\begin{array}{l}P \\
---m\end{array}$ & $\begin{array}{l}\mathrm{S}^{\mathrm{SO}} \mathrm{S}_{4}^{-2} \\
\mathrm{dm}^{-3}-{ }^{---}\end{array}$ & $\mathrm{Al}^{+3}$ & $\mathrm{H}+\mathrm{Al}$ & $\begin{array}{c}\mathrm{K} \\
\mathrm{nmo}\end{array}$ & $\begin{array}{c}\mathrm{Ca} \\
\mathrm{m}^{-3}-\end{array}$ & $\mathrm{Mg}$ & SB & СТC & $\begin{array}{r}\mathrm{M} \\
-----\%\end{array}$ & V \\
\hline 4,1 & 14,1 & 0,1 & 6,4 & 13,5 & 28,3 & 2,2 & 4,4 & 3,7 & 10,4 & 38,6 & 56,5 & 26,8 \\
\hline
\end{tabular}

Fonte: Laboratório de Solos e Tecidos Vegetais, UNOESTE.

Os tratamentos foram constituídos de cindo lâminas de irrigação, sendo: 50, 75, 100, 125 e $150 \%$ da evapotranspiração da cultura (ETc), correspondentes aos tratamentos T1, T2, T3, T4 e T5, respectivamente. A evapotranspiração de referência (ETO) foi determinada pelo método de Penman-Monteith (Equação 01). O coeficiente de cultura utilizado para determinar a evapotranspiração da cultura 
(equação 02) foi de 0,85 (DOORENBOS; KASSAN, 1994). A irrigação foi com turno de rega de 3 dias.

$$
\text { ETo }=\frac{0,408 . s .(R n-G)+\gamma \cdot 900 \cdot U_{2}\left[\frac{\mathrm{es}-\mathrm{ea}}{\mathrm{Td}+273}\right]}{\mathrm{s}+\gamma \cdot\left(1+0,34 \cdot \mathrm{U}_{2}\right)}
$$

$\mathrm{ETc}=\mathrm{ETo} \times \mathrm{kc}$

Em que:

ETO - evapotranspiração de referência $\left(\mathrm{mm} \mathrm{dia}^{-1}\right)$;

$\mathrm{Rn}$ - radiação líquida total diária $\left(\mathrm{MJ} \mathrm{m}^{-2} \mathrm{dia}^{-1}\right)$;

$\gamma$ - constante psicométrica $\left(0,063 \mathrm{kPa}^{\circ} \mathrm{C}^{-1}\right)$;

$\mathrm{T}_{\mathrm{d}}$ - temperatura média diária $\left({ }^{\circ} \mathrm{C}\right)$;

$\mathrm{U}_{2}$ - velocidade do vento $\left(\mathrm{m} \mathrm{s}^{-1}\right)$;

$\mathrm{e}_{\mathrm{s}}$ - pressão de saturação de vapor $(\mathrm{kPa})$;

$\mathrm{e}_{\mathrm{a}}$ - pressão parcial de vapor $(\mathrm{kPa}) ;$ ]

$\mathrm{S}$ - declividade da curva de pressão de vapor $\left(\mathrm{kPa}^{\circ} \mathrm{C}^{-1}\right)$;

ETc - evpoatranspiração da cultura $(\mathrm{mm})$;

Kc - coeficiente da cultura

Os parâmetros avaliados foram: altura quinzenal das plantas (AP), número de folhas quinzenal (NF), massa seca de parte aérea (MSPA), eficiência da água (EUA), comprimento de raiz (CR) e matéria seca de raiz (MSR). A eficiência do uso da água (EUA) foi determinada a partir da razão entre a massa fresca de parte aérea e lâmina total de irrigação (irrigação+precipitação).

Os dados foram submetidos a análise de variância (ANOVA)e as médias dos tratamentos comparadas pelo teste de Scott-Knott ao nível de significância de $5 \%$ Os dados que apresentaram significância foram submetidos a análise de regressão.

\section{RESULTADOS E DISCUSSÃO}

Não se observou diferenças significativas para altura de plantas (Tabela 2), em todos períodos de avaliação em função das laminas de irrigação aplicadas. No período experimental não houve oscilação considerável de temperatura e umidade (Figura 1) o que colaborou para que não ocorresse variação no crescimento das plantas em resposta a variação climática. A temperatura média do ar no período foi de $23^{\circ} \mathrm{C}$ e a umidade relativa média do ar de $65 \%$.

Tabela 2. Altura de planta (AP) da cana-de-açúcar RB 867515 em função de diferentes laminas de irrigação e períodos de avaliação.

\begin{tabular}{cccccc}
\hline \multirow{2}{*}{ LÂMINAS } & \multicolumn{5}{c}{ DIAS APÓS O PLANTIO } \\
\cline { 2 - 6 } & 15 & 30 & 45 & 60 & 75 \\
\hline $50 \%$ & $53,80 \mathrm{a}$ & $54,00 \mathrm{a}$ & $54,00 \mathrm{a}$ & $54,00 \mathrm{a}$ & $59,50 \mathrm{a}$ \\
$75 \%$ & $57,98 \mathrm{a}$ & $58,30 \mathrm{a}$ & $58,30 \mathrm{a}$ & $58,30 \mathrm{a}$ & $62,40 \mathrm{a}$ \\
$100 \%$ & $60,80 \mathrm{a}$ & $60,90 \mathrm{a}$ & $61,20 \mathrm{a}$ & $61,20 \mathrm{a}$ & $61,20 \mathrm{a}$ \\
$125 \%$ & $64,22 \mathrm{a}$ & $64,70 \mathrm{a}$ & $64,70 \mathrm{a}$ & $64,70 \mathrm{a}$ & $70,60 \mathrm{a}$ \\
$150 \%$ & $56,94 \mathrm{a}$ & $57,36 \mathrm{a}$ & $57,36 \mathrm{a}$ & $57,36 \mathrm{a}$ & $68,46 \mathrm{a}$ \\
\hline CV (\%) & 18,28 & 17,92 & 17,92 & 17,92 & 19,09 \\
\hline MG & 58,76 & 59,11 & 59,11 & 59,11 & 64,37 \\
\hline
\end{tabular}

Médias seguidas da mesma letra, minúscula nas colunas, não diferem entre si pelo teste de Skott-Knott, em nível de $5 \%$ de probabilidade.

DAT= dias após o transplantio; $C V=$ coeficiente de variação; $M G=$ média geral 


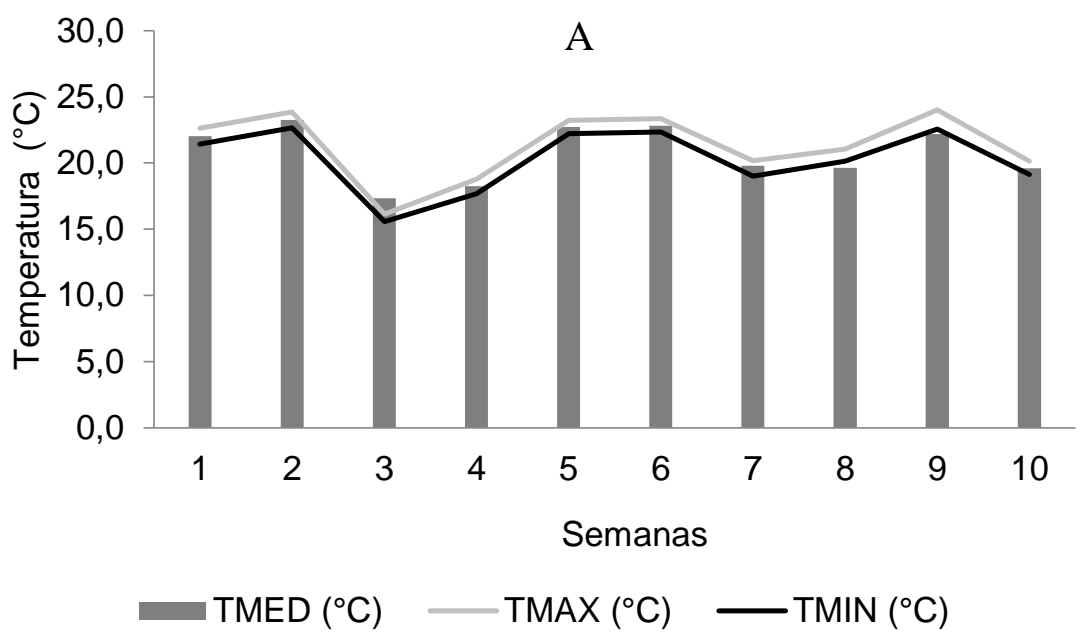

B

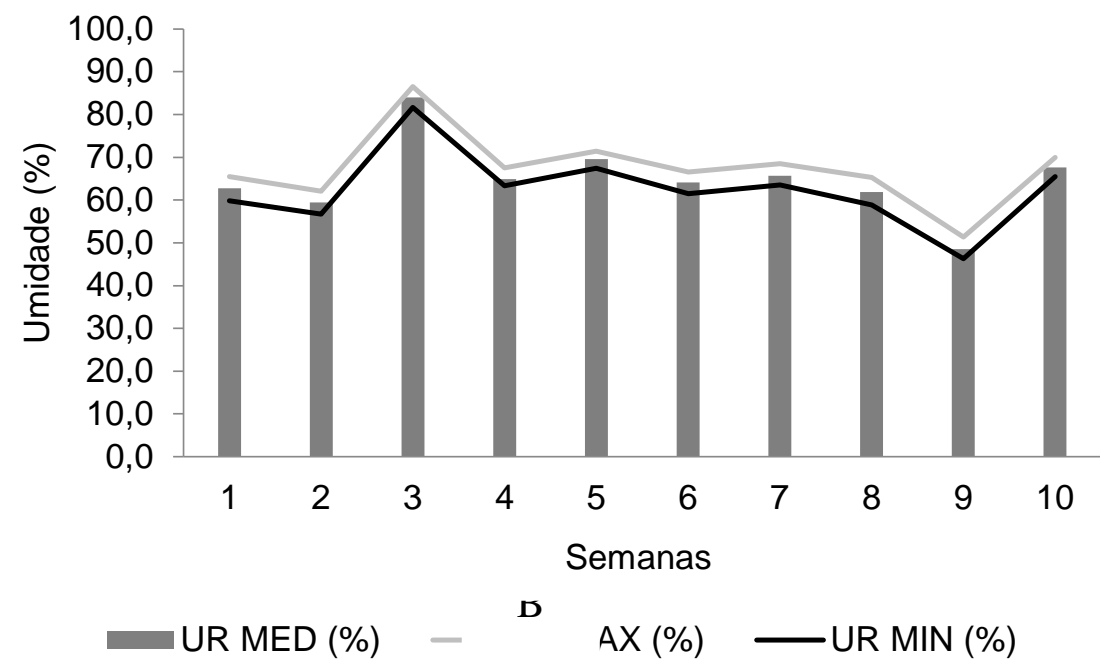

Figura 1. Precipitação semanal (A), Temperatura $\left({ }^{\circ} \mathrm{C}\right)(\mathrm{B})$ e Umidade (\%) (C) durante o período experimental.

Oliveira et al. (2010), observaram que para as variedades RB813804 e RB72454 a altura foi maior quando submetidas a irrigação plena (100\% ETc). Para Oliveira et al. (2014) as cultivares RB 72454 e RB 92579 apresentaram altura do colmo menor para a lâmina de irrigação de $40 \%$ da ETc. Segundo Souza et al. (2015) o crescimento da cana-de-açúcar 791011 tem uma relação direta com a quantidade de água disponível para a cultura.

Na Tabela 3 observou-se que apenas para os 60 dias após o transplantio (DAT) ocorreu diferença entre o número de folhas para as lâminas de irrigação estudadas. A lâmina de irrigação de $75 \%$ ETc propiciou o maior número de folhas. Silva et al. (2015) verificaram que o número de folhas para plantas irrigadas durante a fase I de desenvolvimento (início do crescimento vegetativo) são em torno de 4 a 8 folhas considerando a lâmina de irrigação de $100 \%$ ETc.

De acordo com Inman-Bamber e Smith (2004), o número de folhas pode ser um indicador de déficit hídrico pois a medida que reduz a disponibilidade de água há uma tendência a diminuição da superfície transpirante e dos gastos no metabolismo, senescência das folhas e paralisação do surgimento de folhas. 
Tabela 3. Número de folhas (NF) da cana-de-açúcar submetida às lâminas de irrigação de 50, 75, 100, 125 e $150 \%$ ETc determinado em intervalos de 15 após o plantio.

\begin{tabular}{cccccc}
\hline \multicolumn{5}{c}{ DIAS APÓS PLANTIO } \\
\hline TRATAMENTOS & 15 & 30 & 45 & 60 & 75 \\
\hline $50 \%$ & $3 \mathrm{a}$ & $4 \mathrm{a}$ & $4 \mathrm{a}$ & $5 \mathrm{~b}$ & $5 \mathrm{a}$ \\
$75 \%$ & $4 \mathrm{a}$ & $4 \mathrm{a}$ & $4 \mathrm{a}$ & $6 \mathrm{a}$ & $5 \mathrm{a}$ \\
$100 \%$ & $4 \mathrm{a}$ & $4 \mathrm{a}$ & $5 \mathrm{a}$ & $5 \mathrm{~b}$ & $6 \mathrm{a}$ \\
$125 \%$ & $4 \mathrm{a}$ & $4 \mathrm{a}$ & $5 \mathrm{a}$ & $5 \mathrm{~b}$ & $6 \mathrm{a}$ \\
$150 \%$ & $4 \mathrm{a}$ & $4 \mathrm{a}$ & $5 \mathrm{a}$ & $5 \mathrm{~b}$ & $6 \mathrm{a}$ \\
\hline CV (\%) & 21,7 & 18,6 & 19,2 & 12,9 & 12,9 \\
\hline MG & 4,1 & 3,8 & 4,8 & 5,9 & 5,9 \\
\hline
\end{tabular}

As letras minúsculas comparam as médias na coluna de acordo com o teste Scott-Knott.

DAT $=$ dias após transplantio

$\mathrm{CV}=$ coeficiente de variação.

$M G=$ média geral

Para a massa seca de parte aérea, massa seca de raiz, volume de raiz e comprimento de raiz (Tabela 4) não houve diferença entre as médias obtidas para os tratamentos estudados.

Oliveira et al. (2010), observaram que as variedades RB92579 e SP81-3250 apresentaram a maior produção de matéria seca sob irrigação plena. Farias et al. (2008) concluíram que a maior percentagem de raízes no solo se encontra nas camadas mais superficiais cana irrigada e de sequeiro ao longo do período de cultivo. Diferenças significativas entre as médias dos manejos ocorreram na profundidade de 0 a 15 $\mathrm{cm}$ e 16 a $30 \mathrm{~cm}$ aos 60 DAP. Quando irrigada, a cultura emite mais rapidamente o seu sistema radicular, de forma tal que na primeira camada, a mais úmida, o sistema se encontra ainda superficial com um volume maior de raiz na superfície que na de sequeiro.

Tabela 4. Massa seca de parte aérea (MSPA), Massa seca de raiz (MSR), Volume de raiz (VR) e Comprimento de raiz (CR) da cana-de-açúcar submetida às lâminas de irrigação de 50, 75, 100, 125 e 150\% Etc.

\begin{tabular}{cccc}
\hline TRATAMENTOS & MSPA $(\mathrm{g})$ & MSR $(\mathrm{g})$ & $\mathrm{CR}(\mathrm{cm})$ \\
\hline $50 \%$ & $6,05 \mathrm{a}$ & $15,74 \mathrm{a}$ & $50,50 \mathrm{a}$ \\
$75 \%$ & $6,03 \mathrm{a}$ & $18,19 \mathrm{a}$ & $37,00 \mathrm{a}$ \\
$100 \%$ & $5,06 \mathrm{a}$ & $10,81 \mathrm{a}$ & $52,50 \mathrm{a}$ \\
$125 \%$ & $6,18 \mathrm{a}$ & $15,89 \mathrm{a}$ & $61,75 \mathrm{a}$ \\
$150 \%$ & $6,32 \mathrm{a}$ & $13,97 \mathrm{a}$ & $62,25 \mathrm{a}$ \\
\hline CV $(\%)$ & 38,7 & 24,9 & 17,7 \\
\hline MG & 5,9 & 14,9 & 52,8 \\
\hline
\end{tabular}

As letras minúsculas comparam as médias na coluna de acordo com o teste Skott-Knott.

$\mathrm{CV}=$ coeficiente de variação.

$M G=$ média geral

Na Figura 3 foi possível observar que a lâmina de irrigação de $50 \%$ ETc apresentou a maior eficiência do uso da água, ou seja, ocorreu maior produção com menor quantidade de água. 


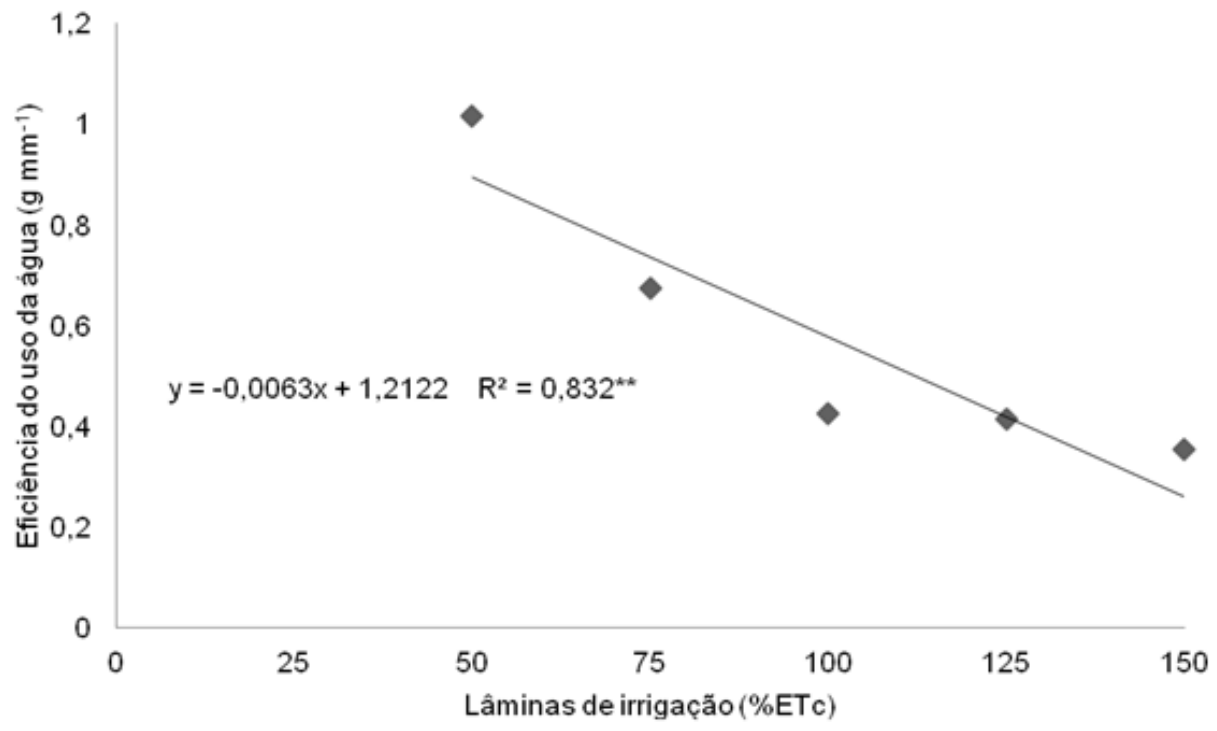

$* *$ significativo a $1 \%$

Figura 2. Eficiência do uso da água para cana-de-açúcar submetidas a lâminas de irrigação de $50,75,100$, 125 e $150 \%$ ETc.

Gava et al. (2010) relataram que as cultivares RB 86 7515, RB 855536 e SP 80-328 apresentaram respostas diferenciadas na eficiência de utilização da água, assim tendo um aumento comparado ao manejo em sequeiro. De acordo com Oliveira et al. (2011) as variedades RB92579 e SP81-3250 de maturação média à tardia se destacaram quanto e à eficiência do uso da água em regime hídrico de1.396,6 $\mathrm{mm}$.

Considerando os parâmetros morfogênico não houve diferenças estatísticas para as lâminas de irrigação estudadas. No entanto, avaliando a relação entre massa seca e lâmina de irrigação aplicada, concluiu-se que 50\% ETc resultou na maior eficiência do uso da água.

\section{REFERÊNCIAS}

BRASIL. Ministério da Agricultura. Cana-deaçúcar. 2014. Disponível em:< http://www.agricultura.gov.br/vegetal/culturas/c ana-de-acucar>. Acesso em: 14 de março2014.

CONAB. Acompanhamento da safra de cana-deaçúcar. Brasília: CONAB, 2014. v.1.

DAMIÃO, J.O.; HERNANDEZ, F.B.T.; SANTOS, G.O.; ZOCOLER, J.L. Balanço hídrico da região de ilha solteira, noroeste paulista. In: CONIRD CONGRESSO NACIONAL DE IRRIGAÇÃO E DRENAGEM, 20. Anais... Uberaba, MG, 6 a 8 de dezembro de 2010.

DANTAS NETTO, J.; FIGUEIREDO, J.L.C.; FARIAS, C.H.A.; AZEVEDO, H.M.; AZEVEDO, C.A.V. Resposta da cana-de-açúcar, primeira soca, a níveis de irrigação e adubação de cobertura.
Revista Brasileira de Engenharia Agrícola e Ambiental, v.10, n.2, p.283-288, 2006. http://dx.doi.org/10.1590/S141543662006000200006 DOOREMBOS, J.; KASSAM, A.H. Efeito da água no rendimento das culturas. Campina Grande: UFPB, 1994. 306p. (Estudos FAO: Irrigação e Drenagem; 33.)

FARIAS, C.H.A. DE; FERNANDES, P.D.; AZEVEDO, H.M. DE; DANTAS NETTO, J. Indices de crescimento da cana-de-açúcar irrigada e sequeiro no estado da Paraíba. Revista Brasileira de Engenharia Agrícola e Ambiental, v.12, p.356362, 2008. http://dx.doi.org/10.1590/S141543662008000400004

GAVA, G.J.C.; KÖLLN, O.T.; URIBE, R.A.M.; TRIVELIN, P.C.O.; CANTARELLA, H. Interação entre água e nitrogênio na produtividade de cana-deaçúcar (Saccharum sp.). In: CRUSCIOL, C.A.C. (Org.). Tópicos em ecofisiolgia da cana-deaçúcar. 1. ed. Botucatu: FEPAF, 2010. v. 1, p. 4966.

INMAN-BAMBER, N.G.; SMITH, D.M. Sugarcane water stress criteria for irrigation and drying off. Field Crops Research, v.89, p.107-122, 2004. http://dx.doi.org/10.1016/j.fcr.2004.01.018 MAGRO, F.J.; TAKAO, G.; CAMARGO, P.E.; TAKAMATSU, S.Y. Biometria em cana-de-açúcar. Piracicaba: ESALQ, 2011.

OLIVEIRA, E.C.A.; FREIRE, F.J.; OLIVEIRA, A.C.; SIMÕES NETO, D.E.; Alexandre Tavares da ROCHA, A.T.; CARVALHO, L.A. Produtividade, eficiência de uso da água e qualidade tecnológica 
de cana-de-açúcar submetida a diferentes regimes hídricos. Pesquisa Agropecuária Brasileira, v.46, n.6, p.617-625, jun. 2011. http://dx.doi.org/10.1590/\$0100-

204X2011000600007

OLIVEIRA, F. M.; AGUILAR, P. B.; TEIXEIRA, M. F. F.; ASPIAZU, I.; MONÇÃO, F. P.; ANTUNES, A. P. S. Características agrotecnólogicas de cana-deaçúcar em diferentes épocas de supressão de irrigação e níveis de adubação. Semina: Ciências Agrárias, Londrina, v. 35, n. 3, p. 1587-1606, $2014 . \quad$ http://dx.doi.org/10.5433/16790359.2014v35n3p1587

OLIVEIRA, E.C.A.; OLIVEIRA, R.I.; ANDRADE, B.M.T.; FREIRE, F.J.; LIRA JÚNIOR, M.A.; MACHADO, P.R. Crescimento e acúmulo de matéria seca em variedade de Cana-de-Açúcar cultivada sob irrigação plena. Revista Brasileira de Engenharia Agrícola e Ambiental, v.14, n.9, p.951-960, 2010. http://dx.doi.org/10.1590/S1415-

43662010000900007

PEREIRA, A.R.; ANGELOCCI, L.R.; SENTELHAS, P.C. Agrometeorologia: fundamentos e aplicações práticas. Guaíba: Agropecuária, 2002. 478 p.

RAMESH, P. Effect of different levels of drought during the formative phase on growth parameters and its relationship with dry matter accumulation in sugarcane. Journal of Agronomy and Crop Science, v.185, p.83-89, 2000. http://dx.doi.org/10.1046/j.1439-

037x.2000.00404.x

SEGATO, S.V.; MATTIUZ, C.F.M.; MOZAMBANI, A.E. Aspectos fenológicos da cana-de-açúcar. In: SEGATO, S.V.; PINTO, A. S.; JENDIROBA, E. NÓBREGA, J.C.M. (org.). Atualização em produção de cana-de-açúcar. Piracicaba: CP 2, 2006. p. 19-36.

SILVA, N.F.; CUNHA, F.N.; TEIXEIRA, M.B.; SOARES, F.A.L.S.; MOURA, L.C. Crescimento vegetativo da cana-de-açúcar submetida a lâminas de irrigação e fertirrigação nitrogenada via gotejamento subsuperficial. Revista Brasileira de Agricultura Irrigada, v.9, n.2, p.79-90, 2015.

SOUZA, J.K.C.; MESQUITA, F.O.; NETO, J.D.; SILVA, M.B.R.; FARIAS, C.H.A.; LIMA, Y.B. Crescimento da cana-de-açúcar submetida a diferentes lâminas de irrigação e adubação com zinco. Agropecuária Científica do Semiárido, v.11, n1, p.114-119, 2015.

WIEDENFELD, R.P.; ENCISO, J. Sugarcane responses to irrigation and nitrogen in semiarid South Texas. Agronomy Journal, v.100, p.665-
671, 2008. http://dx.doi.org/10.2134/agronj2007.0286

Recebido para publicação em 21/09/2015

Revisado em 29/03/2016

Aceito em 13/06/2016 